Ann. Biol. anim. Bioch. Biophys., I968, 8 (3), 339-348.

\title{
RÉPARTITION URINAIRE ET FÉGALE DE L'EXCRÉTION DES CESTROGÈNES CHEZ LA TRUIE ET LA BREBIS
}

\author{
M. TERQUI, P. ROMBAUTS, J. FEVRE \\ avec la collaboration technique de Ginette LEPINE \\ Station centrale de Physiologie animale, \\ Station centrale de Recherches de Nutrition, \\ Centre national de Recherches zootechniques, 78 -Jouy-en-Josas \\ Institut national de la Recherche agronomique
}

\section{SOMMAIRE}

Après injection d'œestrone ou d'œestradiol $17 \beta^{3} \mathrm{H}$ ou ${ }^{14} \mathrm{C}$, les pourcentages d'élimination de la radioactivité, par la voie urinaire et la voie fécale, ont été mesurés chez la Truie et la Brebis.

L'excrétion des œestrogènes se fait à $90 \mathrm{p}$. Ioo par la voie fécale chez les Ovins, alors que chez la Truie l'excrétion est presque uniquement urinaire ( 88 à $99 \mathrm{p} .100$ de la dose injectée). Pour cette espèce, les fractions œstrone et œstradiol ne représentent que 45 à $70 \mathrm{p}$. roo de la radioactivité récupérée. Il est donc certain qu'il existe chez la Truie d'autres métabolites oestrogènes.

\section{INTRODUCTION}

La tendance actuelle pour la détermination des sécrétions d'hormones stéroides consiste à mesurer ces stéroïdes soit dans le sang veineux efférent de la glande, soit dans le sang périphérique. Ces dosages sont réalisables grâce aux techniques très sensibles de double dilution isotopique, de chromatographie en phase gazeuse et de fluorimétrie. La connaissance imparfaite des débits et du volume sanguin total, laisse toutefois une marge d'incertitude dans l'appréciation de la sécrétion. C'est pourquoi, on essaie actuellement de mesurer avec précision la vitesse de production en étudiant parallèlement aux concentrations sanguines la clearance métabolique des sté- 
roïdes à l'aide d'hormones radioactives. Les vitesses de production des stéroïdes hormonaux peuvent être déterminées à partir de l'activité spécifique des métabolites urinaires ou fécaux. Si 1'on désire utiliser des méthodes plus simples que celles des dosages dans le sang, ou des techniques très élaborées, la connaissance des modalités de l'excrétion urinaire des stéroïdes est nécessaire.

Chez certains animaux, les œstrogènes prédominants ne sont pas les mêmes que chez 1'Homme et leur excrétion dans les fèces peut être importante ; ainsi, chez les Bovins, elle est sensiblement équivalente à l'excrétion dans l'urine (MELLIN et ERB, I966), mais chez les Porcins et les Ovins, nos connaissances sont encore très limitées.

WRIGHT (I962) après injection d'œstradiol $I 7 \beta$ à une Brebis ovariectomisée retrouvait, sous forme d'œstradiol I $7 \alpha$ et d'œstrone, $40 \mathrm{p}$. Ioo de la dose injectée dans les fèces, et 2 p. roo dans l'urine. La voie fécale paraît donc très importante chez cette espèce et la faible récupération totale semble indiquer l'existence d'autres métabolites encore inconnus.

Chez une truie ovariectomisée, BowERMAN et al. (Ig64) ne retrouvent dans les fractions œstrone et œestradiol de l'urine que 39 à $60 \mathrm{p}$. Ioo de la dose d'œstradiol injectée. LunAas ( $1963 a$ ) dans des conditions voisines, ne récupère que $50 \mathrm{p}$. roo de l'œestradiol. Cette incertitude sur le sort de la moitié de la dose injectée à ces animaux, ne peut s'expliquer que par l'existence d'autres métabolites d'excrétion des œstrogènes ou par une élimination importante des œestrogènes par les fèces.

L'objet du présent travail est donc l'étude des voies d'excrétion des œstrogènes chez les Porcins et les Ovins. L'isolement et la caractérisation des autres œestrogènes que l'œstrone, chez la Truie, sont actuellement en cours et les résultats seront publiés ultérieurement.

\section{MATÉRIEL ET MÉTHODES}

$$
\text { A. - Matériel }
$$

\section{a) Animaux.}

Chez la Brebis, des mesures ont été effectuées sur le même animal de race Ile-de-France, d'un poids de $65 \mathrm{~kg}$, pendant l'anoestrus saisonnier et au $40^{\mathrm{e}}$ jour de la gestation.

Nous avons utilisé deux truies Large White : la truie $\mathrm{n}^{\circ} 2439$, de $250 \mathrm{~kg}$, dont l'excrétion d'œstrogènes a été étudiée au $16^{\mathrm{e}}$ jour du cycle oestrien, au $27^{\mathrm{e}}$ jour de la gestation et au roo jour de gestation, et la truie 2349 , de $\mathrm{I} 5 \mathrm{okg}$, étudiée au $\mathrm{I}^{\mathrm{er}}$ jour du cycle œstrien et un an après ovariectomie et hystérectomie.

Les animaux étaient placés dans des cages à métabolisme permettant de récolter quantitativement et séparément les urines et les fèces de 24 heures. Après chaque injection, les récoltes d'ex creta ont été poursuivies pendant 10 jours consécutifs.

b) Administration des astrogènes marqués.

L'cestrone $6,7^{-3} \mathrm{H}$ (10 Ci/mmole) et l'œestradiol I $7 \beta 6,7^{-3} \mathrm{H}$ (ro Ci/mmole) provenaient de la firme N. E. N. C. (Boston Mass.) et l'œstrone $4^{-14}(50 \mathrm{~m} \mathrm{Ci} / \mathrm{mmole})$ du Radiochemical Center (Amersham Buck's).

Avant chaque expérience, la pureté radiochimique de ces composés a été contrôlée par chromatographie en couche mince dans le système C de LISBOA (1962) (acétate d'éthyle-cyclohexane 50/50) 
et à l'aide d'un lecteur Packard 720r. Lorsque des impuretés ont été détectées, les œstrogènes marqués sont purifiés par chromatographie sur papier dans le système formamide/orthodichlorobenzène.

Les composés radioactifs, dissous dans une solution de sérum physiologique contenant $2 \mathrm{p}$. 100 d'éthanol, furent injectés par voie intraveineuse.

\section{B. - Méthodes de dosage}

a) Exiraction des astrogènes des fèces.

Pour extraire les oestrogènes des fèces, nous avons adopté la méthode utilisée par ADLERCREUTz (I962) pour les œestrogènes de la bile. Après homogénéisation des fèces de 24 heures, $100 \mathrm{~g}$ de fèces ont été broyés dans $300 \mathrm{ml}$ de méthanol $70 \mathrm{p}$. 100 à l'aide d'un broyeur Ultra-Turrax. La bouillie a été centrifugée, le culot a subi deux autres extractions et les trois surnageants réunis constituent l'extrait à partir duquel la radioactivité est mesurée.

\section{b) Dosage des astrogènes de l'urine de Truie.}

Nous avons utilisé la méthode de Brown avec les modifications décrites dans un article précédent (RombauTs, I962).

Nous avons vérifié que les différentes purifications ne provoquaient pas d'échange isotopique. Pour cela, nous avons ajouté à de l'urine de l'œestrone $6,7^{-2} \mathrm{H}$ et de l'œestrone $4^{-14} \mathrm{C}$ dans un rapport ${ }^{8} \mathrm{H} /{ }^{14} \mathrm{C}$ de $\mathrm{II,2}$; après purification, le rapport de la fraction oestrone était de $\mathrm{II}, \mathrm{I}_{5}$ (moyenne de trois essais). Le rapport ${ }^{3} \mathrm{H} /{ }^{14} \mathrm{C}$ n'étant pas modifié, il n'y a donc pas eu d'échange. Le pourcentage de récupération est de $66 \mathrm{p}$. Ioo pour l'œstrone et de $77 \mathrm{p}$. Ioo pour l'œestradiol. Nous avons remarqué que la fraction " ostradiol " était contaminée par I,I p. 100 d'œestrone. Aussi, pour cette fraction "œestradiol " avons-nous effectué une chromatographie en couche mince (système C de LISBOA) après la chromatographie sur alumine.

\section{c) Mesure de la radioactivité.}

Pour éviter l'écueil d'une perte de radioactivité dans des métabolites inconnus, les mesures ont été effectuées non sur des fractions d'œestrogènes isolés, mais sur l'urine totale et sur l'extrait global de fèces.

Pour mesurer la radioactivité de l'urine et de l'extrait de fèces, nous avons employé le mélange scintillant T 2-I décrit par PatTerson et Greene (1965):

solution $A: \mathrm{I}$ litre de toluène, $4 \mathrm{~g}$ de 2,5 diphényl oxazol (P. P. O.) et $100 \mathrm{mg}$ de $\mathrm{I}, 4$ bis 2-(4 méthyl-5-phényl oxazolyl) benzène (Diméthyl P. O. P. O. P.);

solution B: Triton $\mathrm{X}_{\mathbf{1 0 0}}$ (Touzart et Matignon).

Le mélange scintillant est constitué de deux volumes de la solution A et d'un volume de B. Pour une proportion de $17 \mathrm{ml}$ de ce mélange et $5 \mathrm{ml}$ de solution aqueuse, on obtient un gel stable et homogène de $0^{\circ} \mathrm{C} \grave{a}-5^{\circ} \mathrm{C}$. Les rendements $(r)$ obtenus par un compteur à scintillation Packard 3375 avec ce mélange sont pour :

- le tritium seul, $r=21$ p. 100 (fenêtre $50-1000$, amplification 60 p. 100);

- le carbone 14 seul, $r=82$ p. I00 (fenêtre 50-I 000, amplification 9,75 p. 100);

- le tritium en présence de carbone $14, r=14,5 \mathrm{p}$. roo (fenêtre $50-545$, amplification Ioo p. Ioo, réinjection de ${ }^{14} \mathrm{C}=7 \mathrm{p}$. 100);

- le carbone en présence de tritium, $r=47$ p. 100 (fenêtre 230-1 000, amplification 12,5 p. 100 réinjection de tritium $2 \mathrm{cpm}$ pour $10^{4} \mathrm{dpm}$ ).

Les mesures ont été effectuées à 5 concentrations différentes :

$0,5-$ I $-2-3$ et $4 \mathrm{ml}$ d'urine, complétés à $5 \mathrm{ml}$ par de l'eau;

$0,5-1$ - I,5 - 2 et $3 \mathrm{ml}$ d'extrait fécal également complétés à $5 \mathrm{ml}$.

Les résultats sont la moyenne de ces 5 déterminations.

Pour l'étude des fractions oestrone et œestradiol, nous avons utilisé du toluène contenant $4 \mathrm{~g} / \mathrm{l}$ de P. P. O. et $200 \mathrm{mg} / \mathrm{l}$ de diméthyl P. O. P. O. P.

Les corrections de "Quenching " ont été effectuées, soit par la méthode des dilutions (fig. I), soit par la méthode de la standardisation externe (A.E.S.) (fig. 2). Les deux méthodes ont été comparées et conduisent aux mêmes résultats. Tous les échantillons ont été comptés au moins trois fois ro minutes. 


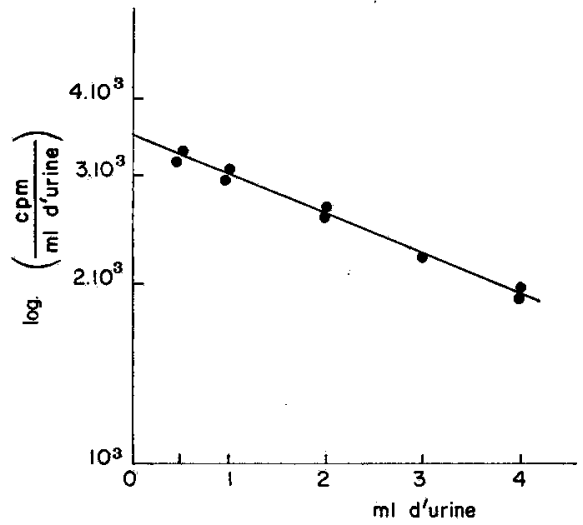

Fig. 1. - Correction de Quenching par la méthode des dilutions

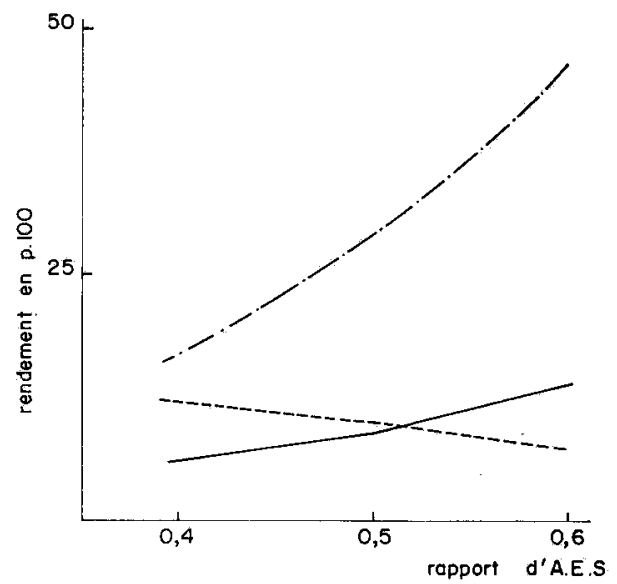

Fig. 2. - Correction de Quenching par standardisation externe

Courbes étalons avec le mélange $\mathbf{T}_{\mathbf{2}-1}$ - Tritium

$--\neg$ Carbone $I_{4}$ dans la voie Tritium

-. - Carbone $\mathrm{I4}$

\section{RÉSULTATS}

\section{A. - Chez la Brebis}

Si l'œstradiol I $7 \alpha$ est le principal métabolite urinaire chez la Brebis, l'cestrone est aussi excrété en quantité importante en fin de gestation (FÈvRE et ROMBAUTS, 1965). Ne disposant pas d'œstradiol I7 $\alpha$ radioactif, nous avons donc injecté à la Brebis, $77 \mu \mathrm{Ci}$ d'œstrone $6,7^{-3} \mathrm{H}$ pendant 1'ancestrus saisonnier et $100 \mu \mathrm{Ci}$ au $40^{\mathrm{e}}$ jour de la gestation.

Les résultats du tableau $\mathrm{I}$, montrent que :

- l'excrétion d'œestrogène est extrêmement rapide puisque 66 et 69 p. 100 de la dose injectée sont récupérés dans les premières 24 heures;

- la voie fécale est prépondérante : 73 et $76 \mathrm{p}$. roo de la dose administrée sont éliminés dans les fèces (tabl. I), ce qui représente 90 et $87 \mathrm{p}$. Ioo de la radioactivité globale retrouvée.

Toutefois, l'élimination urinaire est plus importante que ne le supposait WRIGHT (rg62). Il décrit un pic d'excrétion fécale entre le $5^{\mathrm{e}}$ et le ro jour après l'injection. d'œestradiol I $\beta$ alors que nous avons constaté une très forte excrétion les premières 24 heures, suivie d'une brusque décroissance les jours suivants. Nos résultats sont en accord avec les travaux de GLASCOCK et SMITH (I965) sur la disparition très rapide de l'hexœstrol tritié, dans le sang de la Brebis, et avec ceux de RoBinson (1965) qui constate une décroissance de la radioactivité dans les tissus-cibles chez la Brebis, dès $4 \mathrm{~h} 30$, après l'injection d'œstradiol $I 7 \beta$ marqué. 


\section{TABLEAU I}

\section{Répartition de l'excrétion des astrogènes chez la Brebis}

\begin{tabular}{|c|c|c|c|c|c|c|}
\hline \multirow{3}{*}{$\begin{array}{l}\text { Jours } \\
\text { après l'injection }\end{array}$} & \multicolumn{3}{|c|}{$\begin{array}{l}\text { Brebis en anoestrus } \\
77 \mu \mathrm{Ci} \text { œestrone } 6,7-^{3} \mathrm{H} \text { injectés }\end{array}$} & \multicolumn{3}{|c|}{$\begin{array}{l}\text { Brebis au } 40^{\circ} \text { jour de gestation } \\
100 \mu \mathrm{Ci} \text { œstrone } 6,7-^{\circ} \mathrm{H} \text { injectés }\end{array}$} \\
\hline & \multicolumn{6}{|c|}{ en p. 100 de la radioactivité injectée } \\
\hline & Fèces & Urine & Total & Fèces & Urine & Total \\
\hline $1 \ldots \ldots \ldots$ & 58,4 & 7,4 & 65,8 & 58,1 & 11,1 & 69,2 \\
\hline $2 \ldots \ldots \ldots \ldots \ldots$ & 9,7 & 0,8 & 10,5 & 9,47 & 0,55 & 10,2 \\
\hline $3 \ldots \ldots, \ldots \ldots \ldots$, & $\mathbf{3 , 0}$ & - & 3 & - & - & - \\
\hline $4 \ldots \ldots \ldots \ldots \ldots$ & 2,3 & - & 2,3 & - & - & - \\
\hline $5 \ldots \ldots \ldots \ldots, \ldots$ & 0 & - & - & - & - & - \\
\hline Total.... & 73,4 & 8,2 & 81,6 & 67,57 & 11,65 & 79,4 \\
\hline
\end{tabular}

TABIEAU 2

Répartition de l'excrétion des astrogènes chez la Truie

\begin{tabular}{|c|c|c|c|c|c|}
\hline \multirow{2}{*}{$\begin{array}{c}\text { Truie } \\
\text { no }^{\circ}\end{array}$} & \multirow{2}{*}{ Jour de l'injection } & \multirow{2}{*}{$\begin{array}{l}\text { Stéroïdes marqués, } \\
\text { injectés }\end{array}$} & \multirow{2}{*}{$\begin{array}{c}\text { Dose } \\
\text { injectée } \\
\mu \mathrm{Ci}\end{array}$} & \multicolumn{2}{|c|}{$\begin{array}{l}\text { en p. } 100 \text { de la } \\
\text { radioactivité injectée }\end{array}$} \\
\hline & & & & Fèces & Urine \\
\hline 2349 & 1er jour du cycle & Estrone 6,7-3 $\mathrm{H}$ & 480 & 2,7 & 69,3 \\
\hline 2349 & $\begin{array}{l}\text { Ovariectomisée et } \\
\text { hystérectomisée }\end{array}$ & Estrone 6,7-3 H & 386 & & 88,2 \\
\hline 2439 & $16^{\circ}$ jour du cycle & $\begin{array}{l}\text { Estrone }{ }^{4-14} \mathrm{C} \text { et } \\
\text { Estradiol } 17 \beta \quad 6,7-{ }^{3} \mathrm{H}\end{array}$ & $\begin{array}{l}34,2 \\
145\end{array}$ & & $\begin{array}{l}88 \\
96\end{array}$ \\
\hline 2439 & $27^{\mathrm{e}}$ jour de gestation & $\begin{array}{l}\text { Estrone }{ }^{4-14} \mathrm{C} \text { et } \\
\text { Ostradiol } 17 \beta \quad 6,7-{ }^{3} \mathrm{H}\end{array}$ & $\begin{array}{l}40,6 \\
259\end{array}$ & & $\begin{array}{l}99 \\
99,3\end{array}$ \\
\hline 2439 & $100^{\ominus}$ jour de gestation & $\begin{array}{l}\text { Estrone }{ }^{4-14} \mathrm{C} \text { et } \\
\text { Estradiol } 17 \beta \quad 6,7-{ }^{3} \mathrm{H}\end{array}$ & $\begin{array}{l}25,4 \\
428\end{array}$ & & $\begin{array}{l}80,8 \\
80,7\end{array}$ \\
\hline
\end{tabular}




\section{B. - Chez la Truie}

Si l'œstradiol $I 7 \beta$ est en plus grande quantité que 1'œstrone dans le liquide folliculaire (LuNAAS, I963 b), l'œestrone est le principal métabolite urinaire (LunAAS, I962; ROMBAUTS, Ig62).

Nous avons injecté de l'œstrone $6,7^{3} \mathrm{H}$ ou de $1^{\prime} œ$ œtradiol $17 \beta, 6,7^{-3} \mathrm{H}$ et de 1'œstrone $4^{-14} \mathrm{C}$ aux doses indiquées dans le tableau 2.

Les résultats présentés dans ce tableau montrent que la voie urinaire est la voie essentielle d'élimination. Elle représente 97 à 99 p. 100 de la dose récupérée. Les variations du pourcentage de la radioactivité récupérée sont liées davantage aux erreurs commises dans la détermination de la dose réellement administrée à l'animal, qu'à des variations dues à l'état physiologique des animaux : gestation, cycle.

Les pourcentages de radioactivité retrouvée les plus élevés ont été obtenus en améliorant la technique d'injection.

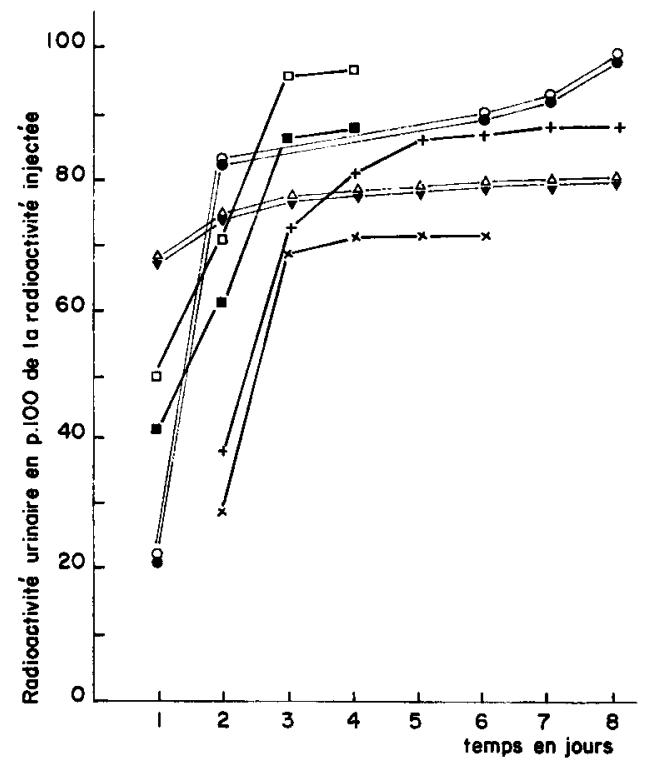

Fig. 3. - Cinétique de l'excrétion urinaire des astrogènes chez la Truie

$-x-$ Truie 2349 , I ${ }^{\text {er }}$ jour du cycle

- + - Truie 2349 , ovariectomisée et hystérectomisée

- $\left.-{ }^{8} \mathrm{H}\right\}$ Truie $2439,16^{\mathrm{e}}$ jour du cycle

=- $\left.-{ }^{34} \mathrm{C}\right\}$ Truie $2439,27^{\mathrm{e}}$ jour de gestation

二 $\left.\Delta{ }^{{ }^{3} \mathrm{C}}\right\}$ Truie 2439, roo jour de gestation

L'excrétion urinaire (fig. 3) est assez rapide puisque 60 à 80 p. Ioo de la dose sont récupérés dans les premières 48 heures.

Puisque nous venons de montrer que l'excrétion fécale était pratiquement inexistante, la seule hypothèse valable pour expliquer la faible récupération obtenue par les autres auteurs dans les fractions œestrone et œestradiol, est l'existence d'autres 


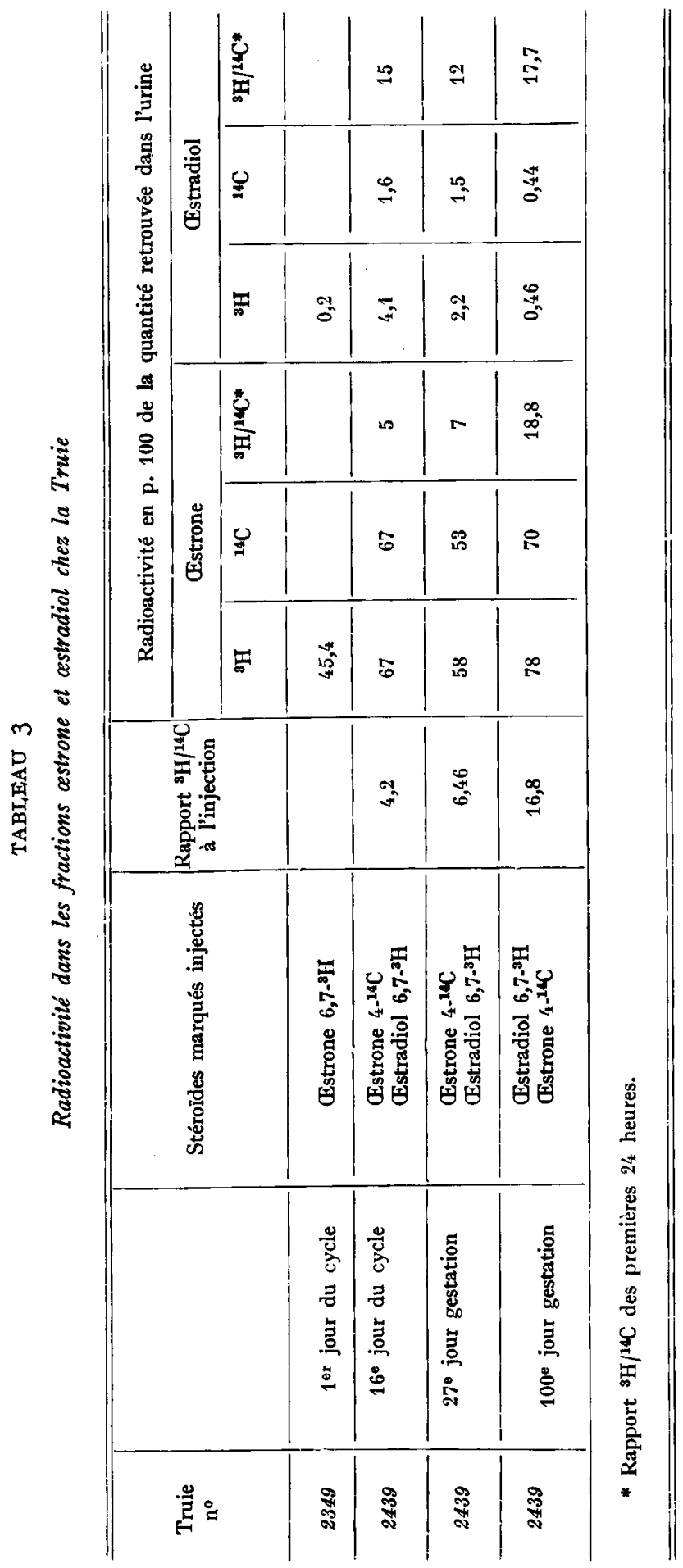


métabolites des œstrogènes. Pour confirmer cette hypothèse, nous avons déterminé le pourcentage de radioactivité dans les fractions œstrone et œstradiol de l'urine (tabl. 3). Il apparaît que :

- 1a radioactivité de la fraction oestrone est la plus importante et celle de la fraction œstradiol est très faible;

- presque tout l'œestradiol I $7 \beta$ initial a été converti en œstrone et en d'autres métabolites; trone ${ }^{14} \mathrm{C}$;

- il s'est formé également de petites quantités d'œestradiol à partir de l'œs-

- la somme de l'cestrone et de l'cestradiol ne représente que 50 à 75 p. Ioo de la radioactivité urinaire. Ces chiffres concordent avec ceux de LUNAAS et de BowERMAN, cités précédemment.

Avant de conclure à la présence d'autres métabolites, nous avons voulu vérifier si l'hydrolyse enzymatique que nous utilisons pour cliver les œestrogènes conjugués était totale.

Pour cela, cinq échantillons de Ioo $\mathrm{ml}$ d'urine provenant d'une truie ayant reçu de 1'œstrone ont été soumis à 1'hydrolyse enzymatique, puis extraits par 3 fois $200 \mathrm{ml}$ d'éther.

L'extrait éthéré contenait 95,6 p. roo de la radioactivité initiale de l'urine et la fraction aqueuse restante, $4,5 \mathrm{p}$. Ioo. Étant donné que le rendement de l'extraction des ostrogènes de l'urine est de $96 \mathrm{p}$. Ioo en moyenne, on peut conclure que l'hydrolyse enzymatique est totale.

La présence d'autres métabolites œstrogènes que l'œstrone et l'œestradiol $I_{7} \beta$ dans l'urine de Truie est donc bien établie. Leur identification est en cours.

\section{DISCUSSION}

Dans les divers stades étudiés, l'excrétion des œstrogènes s'effectue par une voie différente chez les deux espèces étudiées : à 90 p. Ioo par la voie fécale chez la Brebis et à près de Ioo $\mathrm{p}$. Ioo par la voie urinaire chez la Truie. L'analyse des métabolites urinaires est donc très représentative, chez cette dernière espèce, de la sécrétion des œestrogènes. Chez les Ovins, au contraire, il est nécessaire d'effectuer cette analyse dans les fèces et dans l'urine.

Chez la Vache, les travaux de MELLIN et ERB (I966) ont montré un type intermédiaire d'excrétion, puisque 25 à 44 p. Ioo de 1'œstradiol injecté est éliminé dans l'urine.

Pendant l'anœstrus saisonnier et le début de la gestation, l'élimination des œstrogènes chez la Brebis, implique une excrétion quasi totale par la bile. Une dégradation de ces ostrogènes par la flore intestinale ou une incapacité de la paroi intestinale à les réabsorber expliqueraient leur faible élimination rénale.

Chez 1'Homme, une fraction importante des œstrogènes est excrétée par la bile (ADLERCREUTZ, I962) puis réabsorbée par la paroi intestinale et finalement éliminée dans l'urine (BEER et GALLAGHER, I955). 
Il est permis de penser que, chez la Truie, bien que le passage des ostrogènes par la bile ne soit pas démontré, le mécanisme est comparable à celui de l'Homme.

A l'encontre des résultats de MELLIN et ERB chez les Bovins, nous n'avons pas observé, chez la Truie, de grandes différences dans la répartition urinaire et fécale des œstrogènes suivant l'état physiologique : cycle œstrien, gestation ou animal ovariectomisé.

Il existe donc bien des différences très importantes dans les excrétions urinaires et fécales des œestrogènes entre les différentes espèces (Ovins, Porcins ...). De plus, il semble qu'à la lumière des travaux de SchombERg et al. (I965) qui ne récupèrent dans l'urine de Truie que la moitié des métabolites de la progestérone, chaque groupe de stéroïdes puisse avoir des modalités d'excrétion particulières pour une même espèce.

Rȩ̧u pour publication en mars 1968.

\section{SUMMARY}

COMPARATIVE FAECAL AND URINARY EXCRETION OF GESTROGENS IN THE EWE AND SOW

Urinary and faecal radioactivity was measured in the ewe and sow intravenously injected with labelled cestrone and ostradiol I 7 -Beta.

In the ewe, during anœstrus and at day 40 of pregnancy, the main pathway of excretion was the faeces : faecal excretion accounted for $9^{\circ}$ per cent of the recovered radioactivity (table $\mathrm{I}$ ).

In the sow, urinary radioactivity represented 88 to 99 per cent of the injected dose (table 2, fig. 3). The variations in the excretion of radioactivity is not physiologically significant. The cestrone and œstradiol fractions of urine was 45 to 77 per cent of the amount recovered; the œstradiol fraction was only a low percent (table 3 ) and more than a half of the injected cstradiol was converted to cestrone. The presence of other unidentified metabolites is shown in the urine of the sow.

\section{RÉFÉRENCES BIBLIOGRAPHIQUES}

Adlercreutz H., r962. Studies on estrogen secretion in human bile. Acta endocr., suppl., 72.

Bekr C. T., Gallagher T. F., 1955. Excretion of estrogen metabolites by human. I. The fate of small doses of estrone and estradiol $\mathrm{I}_{7} \beta$. J. Biol. Chem., 214, 335-349.

Bowerman A. M., Anderson L. L., Melampy R. M., I964. Urinary estrogens in cycling, pregnant, ovariectomized and hysterectomized gilts. Iowa State J. Sci., 38, 437-445.

Fèvre J., Rombauts P., I966. Étude de l'excrétion urinaire des cestrogènes chez la Brebis pendant la gestation. Ann. Biol. anim. Bioch. Biophys., 6, r65-177.

Glascock, SMith, 1965. Une comparaison de la distribution sanguine et de la vitesse de transport d'un ostrogène à ceux de trois autres constituants sanguins d'un ruminant dans les deux minutes suivant leur injection. II Inier. Symp. C. A. M. I.R. A., 5-8 mai, Gent Belgique, 749-763.

Lisboa B. P., Diczfalusy E., 1962. Separation and characterisation of steroid estrogen by means of thin layer chromatography. Acta Endocr., 40, 60-8I.

LunaAs T., I962. Urinary estrogen levels in the sow during estrous cycle and early pregnancy. J. Reprod. Fert., 4, 13-20.

LunaAs T., I963a. Urinary excretion of estrone in the sow after administration of estradiol $17 \beta$. Acta Endocr., 42, 514-518.

Lunaas T., $1963 b$. Distribution of estrone and estradiol i $7 \beta$ in sow ovaries. Acta Endocr., 44, 529-535. 
Meluin T. N., ERB R. E., 1966. Estrogen metabolism and excretion during the bovine estrous cycle. Steroids, $7,5^{89-606 .}$

Patterson M. S., Green R. C., 1965. Measurement of low energy beta emitters in aqueous solution by liquid scintillation counting of emulsions. Analyt. Chem., 37, 854-857.

Ropinson T. J., r965. Accumulation d'œestradiol et d'hexœestrol tritiés dans le cerveau et quelques autres tissus chez la brebis. Ann. Biol. anim. Bioch. Biophys., 5, 341-35I.

Rombauts P., r962. Excrétion urinaire d'œestrogènes chez la truie pendant la gestation. Ann. Biol.anim. Bioch. Biophys., 2, $151-156$.

Schomberg D. W., Featherson W. R., Merschler R. C., ERB R. E., I964. Excretion of progesterone $4^{-14} \mathrm{C}$ in the sow. J.anim. Sci., 23, I 228.

WRIGHT A. A., 1962. Metabolism of estradiol $17 \beta$ by an ovariectomized ewe. J. Endocr., 24, 29I-297. 